\title{
Revisiones
}

\section{Turnos de trabajo, ¿un factor de riesgo cardiovascular?}

\section{Shift Work, a cardiovascular factor risk?}

\author{
José Santana-Herrera' ${ }^{1,3}$, Tony Alfano ${ }^{2,3}$, Adriana Escobal-Machado ${ }^{1,3}$ \\ 1. Hospital Universitario de Gran Canaria "Dr. Negrín". Las Palmas de Gran Canaria (Gran Canaria). España. \\ 2. Hospital Universitario de Canarias. La Laguna. (Tenerife) España. \\ 3. Unidad Docente de Medicina del Trabajo de Canarias. Las Palmas de Gran Canaria. España.
}

Recibido: 23-01-14

Aceptado: 24-02-14

\author{
Correspondencia \\ José Santana-Herrera \\ Hospital Universitario de Gran Canaria "Dr. Negrín" \\ Servicio de Prevención de Riesgos Laborales \\ Barranco La Ballena $\mathrm{s} / \mathrm{n}$ \\ CP 35010 Las Palmas de Gran Canaria. España. \\ TIf: 928 450665; 928450000 \\ Correo electrónico: jsh@telefonica.net
}

Este trabajo se ha desarrollado dentro del Programa Científico de la Escuela Nacional de Medicina del Trabajo del Instituto de Salud Carlos III en convenio con Unidad Docente de Medicina del Trabajo de Canarias.

Resumen

Objetivos: Describir según la literatura existente si hay evidencia científica de que los turnos de trabajo son un factor de riesgo cardiovascular e identificar las intervenciones en los tipos de turno más efectivas para reducir dichos riesgos.

Material y métodos: Se realizó una búsqueda bibliográfica en 10 bases de datos obteniéndose 13 artículos. El diseño de estos estudios fueron: 7 de intervención, 1 de cohortes, 1 casos control, 3 descriptivos transversales y un estudio piloto. Se agruparon los trabajos según la dirección y velocidad de rotación, ergonomía de los turnos y cambios en el número de turnos analizándose la modificación de los biomarcadores según el tipo de intervención.

Resultados: La muestra total fue de 12.332 trabajadores. El cambio en la dirección de rotación del turno de trabajo de atrás hacia adelante mostró cambios favorables como disminución del nivel de glucosa, TG, Ác. Úrico y TAS. Rotaciones rápidas en sentido horario pueden ser beneficiosas observándose una disminución del colesterol total, relación colesterol total/LDL y un aumento de HDL. Al asociar la dirección y velocidad de rotación no se obtuvieron modificaciones significativas de los biomarcadores. El cambio ergonómico en los turnos disminuyo las alteraciones lipídicas, TA y FC. Finalmente la diabetes mellitus se vio influenciada negativamente cuando cambiamos de tres turnos a dos turnos de trabajo.

Conclusiones: Los artículos publicados sugieren un impacto negativo del trabajo por turnos en el riesgo cardiovascular que puede modificarse favorablemente con cambios en la organización del trabajo (sistema de rotación rápido en sentido horario, turnos flexibles y rotaciones de tres turnos).

Med Segur Trab (Internet) 2014; 60 (234) 179-197

Palabras claves: Organización del horario de trabajo, enfermedades cardiovasculares, biomarcadores, ritmo circadiano, turnos de trabajo. 


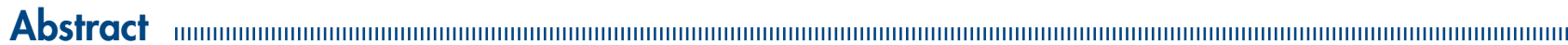

Objective: The aims of this review are to identify the scientific evidence in the association between shift work and cardiovascular risk factor and identify what interventions are more effectives in the different type of shift works to reduce this risk.

Methods: A literature search has been performed in 10 scientific databases, taking into account the level of evidence we select 13 articles. The design of this studies was: 7 of interventions, 1 of cohort, 1 of retrospective cohort, 3 transversals descriptive an 1 pilot study. These covered a diverse range of interventions: changes to the speed, direction, and use of rotation; ergonomics and changes in the numbers to shift work, analyzed the modification of biomarkers by type of intervention.

Results: 12.332 workers were examined. The change in the direction of the shift work from backward to forward shift rotation showed positive changes such as decreased glucose, TG, Ac. Uric and TAS. Rapid clockwise rotation can be beneficial observed a reduced total cholesterol, cholesterol ratio Total/LDL and increase of the HDL. By associating the direction and speed of rotation no significant changes in biomarkers were obtained. The ergonomic change shifts decreased lipid abnormalities, TA and FC. Finally Diabetes Mellitus was negatively influenced when switching from three shifts to two shifts.

Conclusions: The published articles suggest a negative impact of shift work on cardiovascular risk which can be modified favorably to changes in work organization (system fast clockwise rotation, flexible shifts and rotations of three shifts).

Med Segur Trab (Internet) 2014; 60 (234) 179-197

Key words: Work Schedule Tolerance, Cardiovascular Diseases, biomarkers, Circadian Rhythm, Shift Work. 


\section{INTRODUCCIÓN}

La sociedad actual funciona durante las 24 horas del día. Este ritmo es fomentado por el conjunto de las distintas actividades económicas de un país, por lo que el trabajo por turnos es una forma cada vez más común de organización del trabajo.

El tiempo de trabajo es uno de los aspectos de las condiciones de la actividad laboral que tiene una repercusión más directa sobre la vida diaria, además de la salud y el bienestar de los trabajadores.

Si bien la mayoría de las condiciones laborales pueden ser susceptibles de modificación y pueden adaptarse a las necesidades y exigencias de los trabajadores en general, la nocturnidad laboral ya sea permanente (en un turno rotatorio fijo), o no permanente (por ejemplo en turnos rotativos), es una condición laboral inexcusable para millones de trabajadores en todo el mundo. Mientras que la nocturnidad permanente representa una doble alteración (trabajar de noche y dormir de día), la nocturnidad parcial añade como condición laboral extra y diferencial, la rotación de los horarios.

La Directiva Europea 2003/88/CE define al trabajo por turnos como "toda forma de organización del trabajo en equipo por la que los trabajadores ocupan sucesivamente los mismos puestos de trabajo con arreglo a un ritmo determinado, incluyendo el ritmo rotatorio, y que podrá ser de tipo continuo o discontinuo, implicando para los trabajadores la necesidad de realizar un trabajo en distintas horas a lo largo de un periodo dado de días o semanas" ${ }^{1}$.

Según la VII Encuesta Nacional de Condiciones de Trabajo de 2011, un 22,2\% de la población activa trabaja a turnos y un $8,9 \%$ tiene horario nocturno, bien sea en el turno fijo de noche o en jornada de turnos mañana-tarde-noche. Al analizar estos datos, el último informe del Observatorio Estatal de Condiciones de Trabajo, pone de manifiesto, que el porcentaje de este tipo de trabajo ha aumentado en un 5,9\% en los últimos 8 años ${ }^{2}$.

Según Cazamian P. ${ }^{3}$, experto de la OIT, establece que cada quince años de trabajo nocturno se produce un envejecimiento de unos cinco años, y que un tercio de las personas que lo realizan presentan alteraciones físicas incluyendo las enfermedades cardiovasculares.

Las enfermedades cardiovasculares (ECV), son la primera causa de muerte en el mundo y las previsiones de la OMS son que continúen siendo las más prevalentes para el año $2030^{4}$.

Existe una extensa literatura publicada en la última década sobre la asociación entre los turnos de trabajo y los factores de riesgo cardiovascular, demostrando que existe una mayor probabilidad de padecer algún tipo de ECV entre los trabajadores con turnos (rotatorios y nocturnos), en comparación con los que realizan su función de día ${ }^{5-8}$, concluyendo que dichos trabajadores tenían un incremento de ECV del $40 \%$ frente a los trabajadores de día?.

Los factores de riesgo de ECV tradicionales (dislipemia, hipertensión arterial, diabetes mellitus y síndrome metabólico), justifican el 50\% de las patologías cardiovasculares. Sin embargo, mediante nuevos enfoques, las investigaciones se han centrado en buscar otros factores de riesgo entre los que se encuentra el trabajo por turnos.

En el ámbito laboral se han realizado pocos estudios para comparar los distintos turnos de trabajo entre si y analizar cómo se modifican los marcadores de riesgo cardiovascular de estos trabajadores. Se entiende por tanto que la forma de organización del tiempo de trabajo puede convertirse en una condición de "riesgo" cuando hablamos de turnos de trabajo y su relación con el trabajo nocturno. Es por esto que se han sugerido una serie de intervenciones para estudiar los efectos negativos de los turnos sobre la salud del trabajador. 
Los trabajos con un sistema de rotación requieren una adaptación constante de un programa de turnos a otro. De esta forma, el organismo se encuentra en continuo proceso de cambio y de adaptación y desde el punto de vista biológico las funciones fisiológicas varían a lo largo del día.

La mayoría de las funciones del cuerpo humano presentan un ritmo durante el día (concentración de hormonas, lípidos, temperatura, tensión arterial, etc.) que siguen un patrón característico. Esta ritmicidad es controlada por un mecanismo interno o reloj biológico, ubicado en el núcleo supraquiasmático del cerebro, y está influenciado por factores ambientales o sincronizadores, como el trabajo, la actividad, el sueño, las comidas y, en particular, la exposición a la luz.

La falta general de ajuste circadiano es una consecuencia directa del trabajo por turnos. A esto hay que sumarle el hecho de que la mayoría de las personas trabajadoras a turnos tratan de mantener una actividad social y familiar durante el día.

Además, los mecanismos exactos por los que los trabajos a turnos causan enfermedades cardiovasculares no están del todo aclarados. Se han propuesto varios modelos para explicar dicha relación: cambios en los estilos de vida, alteración de los ritmos fisiológicos circadianos, alteración de los biomarcadores de estrés y la interacción entre el trabajo y la familia por lo que se deduce que estos mecanismos son multifactoriales y complejos ${ }^{10}$.

Consciente de esta situación la OIT ha elaborado en el 2011 un informe de expertos sobre la ordenación del tiempo de trabajo, concluye que es necesario un equilibrio aceptable entre las necesidades de los trabajadores (protección de la salud, vida personal y familiar), y los requerimientos de las empresas, sin olvidar por ello las necesidades de la comunidad ${ }^{11}$.

Parece bastante obvio que la vigilancia cuidadosa de salud debe avanzar en paralelo con las acciones correctivas y preventivas en la organización del tiempo de trabajo, y en particular el cambio de programación.

Sin embargo, se debe tener en cuenta que nadie tiene, a priori, la mejor solución, ya que la disposición de las secuencias de cambios se deben adaptar a las exigencias específicas del trabajo, características del personal, las condiciones socio-económicas y los antecedentes culturales de los trabajadores afectados.

Por todo ello nos interesa verificar si los turnos de trabajo en sí mismos son un factor de riesgo cardiovascular. Conocer su efecto y una vez definido esto, proponer medidas preventivas para minimizar este riesgo.

\section{OBJETIVOS}

- Describir según la literatura existente si hay evidencia científica de que los turnos de trabajo son un factor de riesgo cardiovascular.

- Identificar las intervenciones más eficaces para reducir el riesgo cardiovascular.

- Observar a través de intervenciones las modificaciones de los biomarcadores.

- Identificar en las publicaciones revisadas las recomendaciones existentes en relación a la organización del trabajo y proponer actuaciones preventivas.

\section{MATERIAL Y MÉTODOS}

Se realizó una búsqueda bibliográfica de artículos científicos sin límite de fecha de publicación y en cualquier idioma, debido a la escasa bibliografía de esta revisión (última fecha consultada 15/12/2013). Se abordó en bases de datos electrónicas, junto con 
bibliografías, listas de referencias publicadas en distintos soportes, incluyendo manuscritos, tesis e informes encargados por los organismos tanto nacionales como internacionales.

Las bases de datos consultadas fueron: SCOPUS, CISDOC, OVIDSP, IBECS, IBBSST, LILACS, OSH, COCHRANE, MEDLINE (mediante PUBMED). La búsqueda se completó con literatura científica obtenida en el repositorio SciELO, Google y otras fuentes de literatura gris, así como artículos referenciados en los principales trabajos que se incluyeron en el estudio.

Se definieron diferentes estrategias de búsqueda utilizando Descriptores "MeSH, DeCS" y términos libres y ecuaciones de búsqueda que figuran en la tabla I.

Tabla I. Estrategia de búsqueda

\begin{tabular}{|c|c|}
\hline Bases de datos & Descriptores y ecuación de búsqueda \\
\hline & Work Schedule Tolerance* AND Cardiovascular Diseases \\
\hline SCOPUS & Work Schedule Tolerance AND biomarkers \\
\hline CISDOC & Work Schedule Tolerance AND Circadian Clocks \\
\hline OVIDSP & Work Schedule Tolerance AND Circadian Rhythm \\
\hline IBECS & Work Schedule Tolerance AND Intervention Studies \\
\hline IBBSST & Work Schedule Tolerance AND Cardiovascular Diseases AND biomarkers \\
\hline LILACS & Work Schedule Tolerance AND Cardiovascular Diseases AND Circadian Clocks \\
\hline $\mathrm{OSH}$ & Work Schedule Tolerance AND Cardiovascular Diseases AND Circadian Rhythm \\
\hline COCHRANE & Work Schedule Tolerance AND Cardiovascular Diseases AND Intervention Studies \\
\hline MEDLINE & Work Schedule Tolerance AND Cardiovascular Diseases AND hypertension \\
\hline \multirow[t]{24}{*}{ SCIELO } & Work Schedule Tolerance AND Cardiovascular Diseases AND lipids \\
\hline & Work Schedule Tolerance AND Cardiovascular Diseases AND lipoproteins \\
\hline & Work Schedule Tolerance AND Cardiovascular Diseases AND blood presure \\
\hline & Work Schedule Tolerance AND Cardiovascular Diseases AND HbA1c \\
\hline & Work Schedule Tolerance AND Cardiovascular Diseases AND cortisol \\
\hline & Work Schedule Tolerance AND Cardiovascular Diseases AND adrenaline \\
\hline & Work Schedule Tolerance AND Cardiovascular Diseases AND glucocorticoids \\
\hline & Shift Work** AND Cardiovascular Diseases \\
\hline & Shift Work AND biomarkers \\
\hline & Shift Work AND Circadian Clocks \\
\hline & Shift Work AND Circadian Rhythm \\
\hline & Shift Work AND Intervention Studies \\
\hline & Shift Work AND Cardiovascular Diseases AND biomarkers \\
\hline & Shift Work AND Cardiovascular Diseases AND Circadian Clocks \\
\hline & Shift Work AND Cardiovascular Diseases AND Circadian Rhythm \\
\hline & Shift Work AND Cardiovascular Diseases AND Intervention Studies \\
\hline & Shift Work AND Cardiovascular Diseases AND hypertension \\
\hline & Shift Work AND Cardiovascular Diseases AND lipids \\
\hline & Shift Work AND Cardiovascular Diseases AND lipoproteins \\
\hline & Shift Work AND Cardiovascular Diseases AND blood presure \\
\hline & Shift Work AND Cardiovascular Diseases AND HbA1c \\
\hline & Shift Work AND Cardiovascular Diseases AND cortisol \\
\hline & Shift Work AND Cardiovascular Diseases AND adrenaline \\
\hline & Shift Work AND Cardiovascular Diseases AND glucocorticoids \\
\hline
\end{tabular}

En Google la búsqueda se realizó en lenguaje natural mediante las palabras Work Schedule Tolerance, Shift work, Cardiovascular Diseases, biomarkers, Circadian Rhythm, Intervention Studies con diferentes combinaciones de las mismas.

\section{CRITERIOS DE INCLUSIÓN Y EXCLUSIÓN}

Nuestra revisión procuró identificar todos los estudios empíricos que relacionan el trabajo a turnos y las enfermedades cardiovasculares, extrayendo los que habían comparado los efectos de los distintos turnos rotatorios. 
1. Criterios de inclusión:

- Publicaciones cuyas intervenciones se realicen entre los trabajadores a turnos.

- Dentro de los trabajos de intervención, aquellos que utilizan como medida de exposición los biomarcadores de riesgo cardiovascular.

- Trabajos que aun no siendo de intervención (descriptivos, cohortes, casos controles, estudios piloto), comparan dos tipos diferentes de turnos rotatorios mediante biomarcadores.

2. Criterios de exclusión:

- Los estudios que utilizando marcadores biológicos de riesgo cardiovascular no comparen dos sistemas de rotación diferentes.

- Las publicaciones con grupo de control de trabajadores de día que comparen un único turno rotatorio.

- Los trabajos de intervención que valoren la calidad del sueño o las alteraciones del sueño-vigilia.

- Los trabajos de intervención cualitativos.

Una vez seleccionados los títulos y resúmenes de los artículos, se evaluaron de forma independiente por cada investigador, dirimiéndose las controversias mediante revisión conjunta y consenso sobre la pertinencia de su inclusión en la lectura sistemática, siguiendo los criterios descritos anteriormente.

Posteriormente se procedió a la recuperación de artículos a texto completo, a través de la biblioteca de la Escuela Nacional de Medicina del trabajo.

A partir de la estrategia de búsqueda se obtuvo una colección con un total de 875 artículos, que se detallan en la tabla II, de la que fueron eliminados los duplicados y a los que se le aplicó una selección de pertinencia, quedando un total de 547 artículos.

Tabla II. Resultados obtenidos

\begin{tabular}{lcc}
\hline \multicolumn{1}{c}{ Bases de datos } & $\begin{array}{c}\text { Artículos } \\
\text { recuperados }\end{array}$ & $\begin{array}{c}\text { Artículos } \\
\text { seleccionados }\end{array}$ \\
\hline SCOPUS & 128 & 1 \\
CISDOC & 23 & 0 \\
OVIDSP & 115 & 1 \\
IBECS & 6 & 0 \\
IBBSST & 0 & 0 \\
LILACS & 22 & 0 \\
OSH & 98 & 1 \\
COCHRANE & 32 & 0 \\
MEDLINE & 653 & 10 \\
SCIELO & 18 & 0 \\
TOTAL & 875 & 13 \\
\hline
\end{tabular}

Posteriormente, tras la aplicación de los criterios de inclusión y exclusión, quedaron finalmente seleccionados para el trabajo 13 artículos que cumplían todas las condiciones: 


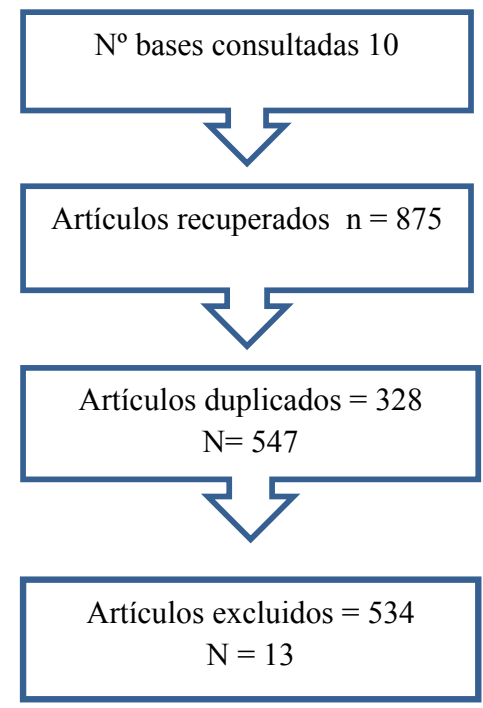

\section{RESULTADOS}

En la tabla III se presentan las principales características de los 13 artículos que componen el estudio, tales como: nombre del autor principal, título, descripción del diseño del estudio, tamaño de la población estudiada, resultados y conclusiones principales del estudio.

Tabla III. Tabla de resultados sistematizados

\begin{tabular}{|c|c|c|c|c|c|}
\hline Autor & Muestra & $\begin{array}{l}\text { Tipo de } \\
\text { estudio }\end{array}$ & Intervención & Variables & Resultados \\
\hline $\begin{array}{l}\text { Orth- } \\
\text { Gomer }^{12} \\
1982\end{array}$ & $\begin{array}{c}\text { Policías } \\
45 \hat{0}\end{array}$ & $\begin{array}{l}\text { Estudio de } \\
\text { intervención }\end{array}$ & $\begin{array}{l}\text { Dirección de } \\
\text { rotación: } \mathrm{CCW} \\
\text { a CW. }\end{array}$ & $\begin{array}{l}\text { TG, ácido úrico, } \\
\text { colesterol, } \\
\text { glucosa, TA, FC } \\
\text { Catecolaminas, }\end{array}$ & $\begin{array}{l}\downarrow \text { glucosa }(\mathrm{t}=4,65, \mathrm{p}<0,001) \\
\downarrow \text { TG }(\mathrm{t}=2,95, \mathrm{p}=0,005) \\
\downarrow \text { Ac. Úrico }(\mathrm{t}=1,68, \mathrm{p}=0,100) \\
\downarrow \text { TAS }(\mathrm{t}=2,52, \mathrm{p}<0,05) \\
\uparrow \text { Epinefrina }(\mathrm{t}=1,91, \mathrm{p}=0,033) \\
\uparrow \text { Norepinefrina }(\mathrm{t}=2,19, \mathrm{p}=0,05)\end{array}$ \\
\hline $\begin{array}{l}\text { Costa }^{13} \\
1993\end{array}$ & $\begin{array}{l}\text { Enfermeras } \\
15 q\end{array}$ & $\begin{array}{l}\text { Estudio de } \\
\text { Intervención }\end{array}$ & $\begin{array}{l}\text { Sistema de } \\
\text { rotación CWr. } \\
\text { T-MM-T-NN-LL } \\
\text { MM-TT-NN-LL }\end{array}$ & $\begin{array}{l}\text { TA, FC, Cortisol } \\
\text { Catecolaminas } \\
6 \text { sulfato } \\
\text { melatonina } \\
(6-S M)\end{array}$ & $\begin{array}{l}\uparrow \text { Adrenalina } \mathrm{F}=5,245 \mathrm{P}=0,004 \\
\uparrow \mathrm{N} / \mathrm{A}: \mathrm{F}=62.89 ; \mathrm{p}<0.001 \\
=\text { Cortisol } \\
=\mathrm{TA}, \mathrm{FC} \\
=6-\mathrm{SM}\end{array}$ \\
\hline $\begin{array}{l}\text { Kecklund }^{14} \\
1994\end{array}$ & $\begin{array}{l}\text { Trabajadores de } \\
\text { Industria } \\
72 \hat{0}\end{array}$ & $\begin{array}{l}\text { Estudio de } \\
\text { Intervención }\end{array}$ & $\begin{array}{l}\text { Velocidad de } \\
\text { rotación: lento } \\
\text { a rápido }\end{array}$ & TA y lípidos. & $\begin{array}{l}\downarrow \text { Col. total }=20 \% \text { a } 11 \%(\mathrm{p}<0.1) \\
\uparrow \mathrm{HDL} / \downarrow \mathrm{LDL} / \mathrm{HDL} \\
\downarrow \mathrm{TA}\end{array}$ \\
\hline $\begin{array}{l}\text { Bøggild }^{15} \\
2001\end{array}$ & $\begin{array}{l}\text { Enfermeras y } \\
\text { auxiliares de } \\
\text { enfermería } \\
101 \%\end{array}$ & $\begin{array}{l}\text { Estudio de } \\
\text { intervención }\end{array}$ & $\begin{array}{l}\text { Avance rápido } \\
\text { giro } \\
\text { Regularidad } \\
\text { turnos } \\
\text { Retraso inicio } \\
\text { mañana } \\
\end{array}$ & $\begin{array}{l}\text { Colesterol total, } \\
\text { HDL, LDL y TG. }\end{array}$ & $\begin{array}{l}\downarrow 8 \% \text { Colesterol total }(\mathrm{p}=0,184) \\
\uparrow 9 \% \text { HDL }(\mathrm{p}=0,001) \\
\downarrow 15 \% \mathrm{LDL}(\mathrm{p}=0,003) \\
\downarrow \mathrm{C} \text { total } / \mathrm{HDL}(\mathrm{p}=0,00)\end{array}$ \\
\hline $\begin{array}{l}\text { Nesthus }^{16} \\
2001\end{array}$ & $\begin{array}{l}28 \\
\text { Controladores } \\
\text { aéreos de } \\
\text { Estados Unidos } \\
(12 \bigcirc \text { y } 16 \text { + })\end{array}$ & $\begin{array}{l}\text { Estudio de } \\
\text { Intervención }\end{array}$ & $\begin{array}{l}\text { Dirección de } \\
\text { rotación CW / } \\
\mathrm{CCW}\end{array}$ & Temperatura & $\begin{array}{l}\downarrow \text { Amplitud: } \mathrm{F}(1,21)=14: 62, \\
\mathrm{P}<0.05 \\
\text { CCW: }\left(\mathrm{M}=0,3^{\circ} \mathrm{C}\right) ; \mathrm{CW}:\left(\mathrm{M}=0,5^{\circ} \mathrm{C}\right) \\
\downarrow \text { Acrofase } \mathrm{F}(1,21)=4,43, \mathrm{p}<0,05 \\
\text { CCW }(\mathrm{M}=18,08) ; \mathrm{CW}(\mathrm{M}=16,44)\end{array}$ \\
\hline
\end{tabular}




\begin{tabular}{|c|c|c|c|c|c|}
\hline Autor & Muestra & $\begin{array}{l}\text { Tipo de } \\
\text { estudio }\end{array}$ & Intervención & Variables & Resultados \\
\hline $\begin{array}{l}\text { De Valck } \\
2007\end{array}$ & $\begin{array}{l}\text { Trabajadores } \\
\text { planta química } \\
36 \pi\end{array}$ & $\begin{array}{l}\text { Estudio de } \\
\text { intervención }\end{array}$ & $\begin{array}{l}\text { Dirección } \\
\text { y velocidad } \\
\text { rotación } \\
\text { CWr / CCW } 1\end{array}$ & cortisol & $\begin{array}{l}=\text { cortisol } \\
\mathrm{CW}=4,85(\mathrm{SE} 0,41) \mathrm{mg} / \mathrm{l} \\
\mathrm{CCW}=4,91(\mathrm{SE} 0,41) \mathrm{mg} / \mathrm{l}\end{array}$ \\
\hline $\begin{array}{l}\text { Vittasalo }^{18} \\
2008\end{array}$ & $\begin{array}{l}\text { Trabajadores de } \\
\text { línea aérea } \\
84 \hat{0}\end{array}$ & $\begin{array}{l}\text { Estudio de } \\
\text { Intervención }\end{array}$ & $\begin{array}{l}\text { Dirección de } \\
\text { rotación: } \\
\mathrm{CCW} 1 \text { / CWr. }\end{array}$ & $\begin{array}{l}\text { TA, FC, } \\
\text { colesterol total, } \\
\text { HDL, LDL, TG, } \\
\text { glucosa, } \\
\text { HbA1c, PCR } \\
\end{array}$ & $\begin{array}{l}\downarrow \text { TAS } \geq 6 \text { mmHg turnos } \\
\text { flexibles } \\
\downarrow \text { FC turnos flexibles } \\
\uparrow \text { TAS } 2.5 \mathrm{mmHg} \text { en } \mathrm{CW} \mathrm{r}\end{array}$ \\
\hline $\begin{array}{l}\text { Morikawa }^{19} \\
2005\end{array}$ & $\begin{array}{l}\text { Trabajadores } \\
\text { japoneses } \\
\text { fábrica de } \\
\text { plástico } \\
2860 \hat{\sigma}\end{array}$ & $\begin{array}{l}\text { Estudio de } \\
\text { cohorte }\end{array}$ & $\begin{array}{l}\text { Rotación } 3 \\
\text { turnos } \\
\text { Rotación } 2 \\
\text { turnos con } \\
\text { CCW. }\end{array}$ & Diabetes: HbA1c & $\begin{array}{l}\uparrow \mathrm{DM} \\
2 \text { turnos RR } 2.01(1.00 \text { a } 4.34) \\
3 \text { turnos RR } 1.61(0.88 \text { a } 2.97)\end{array}$ \\
\hline $\begin{array}{l}\text { Oyama }^{20} \\
2012\end{array}$ & $\begin{array}{l}\text { Trabajadores } \\
\text { japoneses } \\
\text { industria } \\
\text { química } \\
6413 \hat{0} \\
\end{array}$ & $\begin{array}{l}\text { Estudio de } \\
\text { casos y } \\
\text { controles } \\
\text { retrospec- } \\
\text { tivo }\end{array}$ & $\begin{array}{l}\text { Rotación } 2 \\
\text { turnos. } \\
\text { Rotación } 3 \\
\text { turnos con CCW. }\end{array}$ & Diabetes: HbA1c & $\begin{array}{l}\uparrow \uparrow \mathrm{DM} \\
2 \text { turnos- OR } 3,02 \text { ( } 2,50 \mathrm{a} \\
3,66) \text {. } \\
3 \text { turnos-OR } 1,93(1,61 \text { a } 2,32) .\end{array}$ \\
\hline $\begin{array}{l}\text { Vangelova }^{21} \\
2008\end{array}$ & $\begin{array}{l}25 \text { ingenieros } \\
\text { de sonido } 8 \overbrace{}^{\lambda} \\
\text { y } 17 q\end{array}$ & $\begin{array}{l}\text { Estudio } \\
\text { Transversal }\end{array}$ & $\begin{array}{l}\text { Dirección } \\
\text { rotación } \mathrm{CWr} / \\
\mathrm{CCWr}\end{array}$ & cortisol & $\begin{array}{l}\uparrow \text { Cortisol } \\
\text { Turno de rotación } \mathrm{CCW} \\
(\mathrm{F}(3,66)=6.046, \mathrm{p}=0.001)\end{array}$ \\
\hline $\begin{array}{l}\text { Puttonen }^{22} \\
2012\end{array}$ & $\begin{array}{l}1.811 \\
\text { trabajadores } \\
\text { compañía } \\
\text { aérea. } \\
\left(1009 \partial^{\Uparrow} \text { y } 802 \text { ○) }\right.\end{array}$ & $\begin{array}{l}\text { Estudio } \\
\text { Transversal }\end{array}$ & $\begin{array}{l}\text { Ex trabajador } \\
\text { turnos } \\
\text { Trabajador } 2 \\
\text { turnos Turno } \\
\text { de noche }\end{array}$ & $\begin{array}{l}\text { Síndrome } \\
\text { metabólico (SM) }\end{array}$ & $\begin{array}{l}\uparrow \text { SM Ex trabajadores: } \\
\text { IDF: OR } 2,13(1,35 \text { a } 3,37) \\
\text { NCEP: OR } 1.83(1,13 \text { a } 2,96) \\
=2 \text { turnos: } \\
\text { IDF: OR } 0,93(2,24 \text { a } 1,48)\end{array}$ \\
\hline $\begin{array}{l}\text { Mauss }^{23} \\
2013\end{array}$ & $\begin{array}{l}\text { Compañía } \\
\text { Industrial } \\
\text { Alemana } \\
765 \lambda\end{array}$ & $\begin{array}{l}\text { Estudio } \\
\text { transversal }\end{array}$ & $\begin{array}{l}\text { Rotaciones de } \\
3 \text { o } 4 \text { turnos } \\
\text { sentido CCW }\end{array}$ & $\begin{array}{l}\text { SM } \\
\text { FC }\end{array}$ & $\begin{array}{l}\uparrow \mathrm{SM} \\
3 \text { turnos OR } 2,56(1,38 \text { a } 4, \\
75) . \\
4 \text { turnos OR } 1,22(0,73 \text { a } 2,05) \\
\uparrow \text { FC OR } 2,2(1,04 \text { a } 4,63) .\end{array}$ \\
\hline $\begin{array}{l}\text { Kanterman }{ }^{24} \\
2013\end{array}$ & $\begin{array}{l}{ }^{4} \text { Fábrica de } \\
\text { Acero } \\
77 \hat{0}\end{array}$ & $\begin{array}{l}\text { Estudio } \\
\text { piloto. }\end{array}$ & $\begin{array}{l}\text { Dirección de } \\
\text { rotación } \\
\mathrm{CWr} / \mathrm{CCW} 1\end{array}$ & $\begin{array}{l}\text { FC, TA } \\
\text { Velocidad onda } \\
\text { del pulso (VOP). }\end{array}$ & $\begin{array}{l}=\mathrm{VOP} \\
=\mathrm{FC} \\
=\mathrm{TA}\end{array}$ \\
\hline
\end{tabular}

Abreviaturas: CCW: Sentido antihorario o en contra de las agujas del reloj. CW: Sentido horario o a favor de las agujas del reloj. CCWl/r: Sentido antihorario lento/rápido. CWl/r: Sentido horario lento/rápido.

Desde el punto de vista del análisis metodológico el tipo de diseño de los estudios incluidos en la revisión fueron: 7 estudios de intervención, 1 estudio de cohortes, 1 estudios de casos-control, 3 estudios descriptivos transversales y 1 estudio piloto.

Se analizan siete artículos de intervención ${ }^{12-18}$ a nivel organizativo, de los cuales 5 están aleatorizados ${ }^{12,14-16,21}$. Estos cubren una amplia gama de intervenciones: cambios en la velocidad y dirección de rotación, cambios en número de noches; comienzo más tarde, cambios en el trabajo de fin de semana; auto programación de los turnos o combinación de los anteriormente mencionados. El tamaño de las muestras oscila desde los 15 hasta 172 individuos con tiempo de seguimiento que van desde las 4 semanas hasta los tres años.

Del total de los artículos revisados, ocho tenían grupo control ${ }^{14,15,18-20,22-24}$ que generalmente fueron reclutados de diferentes secciones de la misma organización y seis estaban ajustados por factores de confusión ${ }^{14,15,18-20,22}$ : edad, sexo, experiencia de trabajo por turnos, los factores de estilo de vida, o el contenido del trabajo. La mayoría de los estudios se realizaron en poblaciones relativamente homogéneas (por ej enfermeras, controladores aéreos, etc), pero ninguno presenta resultados diferenciados por edades y solamente uno por nivel socioeconómico ${ }^{19}$. 
En cuanto al género tres investigaciones incluyen ambos $\operatorname{sexos}^{16,21,22}$ y dos son realizadas solo con mujeres ${ }^{13,15}$.

Biomarcadores: Dentro de la diversidad de biomarcadores analizados, dos trabajos se centran en la alteración de los lípidos ${ }^{14,15}$ y de la combinación de estos con otros marcadores (glucosa, ácido úrico, la presión arterial, la excreción urinaria catecolaminas), tenemos los trabajos de Orth-Gomer K. et al. ${ }^{12}$ y Viitasalo K. et al. ${ }^{18}$. El cortisol como medidor de estrés está presente en tres de los trabajos ${ }^{13,17,21}$. La Temperatura como marcador de alteración del ritmo circadiano fue analizado en un estudio ${ }^{16}$. En los trabajos de Morikawa Y. et al. ${ }^{19}$ y Oyana I. et al. ${ }^{20}$ se utilizó como marcador de diabetes la hemoglobina glicosilada y en otros dos estudios analizaron el síndrome metabólico ${ }^{22,23}$ entre los trabajadores a turnos. Kantermann T. et al. ${ }^{24}$ utilizó la onda venosa del pulso como marcador de riesgo arteriosclerótico.

Tipos de intervención: Los cambios en la rotación de turnos (dirección y/o velocidad) fueron comparados en siete de los trabajos ${ }^{12,14,16-18,21,24}$. Cambios en la regularidad de los turnos, menos noches consecutivas, reducción de la jornada laboral o más fines de semana libre se ha estudiado en los trabajos de Costa G. et al. ${ }^{13}$ y Bøggild H. et al. ${ }^{15}$ Rotaciones de dos, tres o cuatro turnos fue analizado en cuatro trabajos ${ }^{19,20,23,22}$.

Resumen de las principales características de los artículos revisados:

\section{Artículos de intervención}

\section{Orth-Gomer K. et al. ${ }^{12}$}

Evaluó los efectos de un nuevo calendario de rotación en 45 policías masculinos durante cuatro semanas que fueron divididos en dos grupos, 4 semanas en su horario habitual (en sentido contrario de las agujas del reloj), y 4 semanas con el nuevo horario (con rotación a favor de las agujas del reloj o hacia la derecha).

Antes de iniciar el experimento se evaluaron los niveles de referencia de los factores de riesgo coronario, se estimó la calidad del sueño nocturno, y las tasas de excreción de catecolaminas en reposo.

No se encontraron diferencias estadísticamente significativas en las mediciones basales de ambos grupos de policías. El peso relativo del cuerpo, el colesterol sérico en ayunas, triglicéridos, glucosa y ácido úrico se encontraban dentro de los límites normales y fueron muy similares en los dos grupos. La PA sistólica y diastólica, así como los niveles de epinefrina y norepinefrina no difirió significativamente entre los dos grupos de estudio.

Tras la intervención la glucosa sérica $(\mathrm{t}=4,65 ; \mathrm{p}<0,001)$ y los triglicéridos $(\mathrm{t}=2,95$; $\mathrm{p}=0,005)$ fueron significativamente inferiores durante la rotación en sentido horario en comparación con la rotación en sentido antihorario, no estando afectado el colesterol $(\mathrm{t}=1,33 ; \mathrm{p}=0,191)$. Los niveles de ácido úrico en suero también fueron más bajos durante la rotación en sentido horario $(t=1,68 ; \mathrm{p}=0,100)$, pero la diferencia no alcanzó significación estadística.

La media de la presión arterial sistólica fue significativamente menor a favor de las agujas del reloj $(111.6 \pm 11.2 \mathrm{~mm} \mathrm{Hg}$, en comparación con la rotación en sentido antihorario $(115,6 \pm 10,8 \mathrm{mmHg}, \mathrm{t}=2,52 ; \mathrm{p}<0,05)$. La media de la presión arterial diastólica no difirió significativamente entre los dos horarios $(75,2 \pm 9,9 \mathrm{~mm} \mathrm{Hg}$ y 75,8 \pm 8,3 $\mathrm{mm} \mathrm{Hg} ; \mathrm{t}=0,41)$.

Las tasas de excreción de catecolaminas se incrementaron durante las primeras semanas de rotación a favor de las agujas del reloj, pero cayeron hacia el final del período de observación. Durante la rotación en sentido antihorario, sin embargo, las tasas de excreción de ambos compuestos aumentaron. Sólo en la última evaluación la diferencia entre los horarios alcanzó significación estadística $(t=1,91$ para la epinefrina, $\mathrm{p}=0,033$; si se aplica una prueba $\mathrm{t}$ de una cola, $\mathrm{y} t=2,19$ para la norepinefrina; $\mathrm{p}=0,035$ ). 


\section{Costa G. et al. ${ }^{13}$}

Quince enfermeras, de edades comprendidas entre 21 y 29 años empleadas de una unidad de cuidados intensivos, fueron examinadas con el ánimo de evaluar su adaptación psicofísica a uno de los sistemas de turnos más utilizados, los sistemas de turnos rápidamente rotativos 2-2-2-2 (2 Mañanas-2 Tardes-2 Noches-2 Libres), con tres turnos de 8 horas rotativos cada dos días.

Estos fueron modificados con principios ergonómicos y en relación a las características del trabajo, acortándose los turnos de día de 8 a 7 horas en reconocimiento de mayor carga de trabajo, retraso en el comienzo en el turno de mañana para permitir un sueño más largo y la inclusión dos turnos de noche consecutivos de 10 h de duración, siempre seguidos por 2 días de descanso. El primero de los dos turnos de la tarde fue trasladado al inicio del ciclo de cambio con el fin de crear un período de descanso más largo (T-MMT-NN-LL en lugar de MM-TT-NN-LL).

Durante el turno se midieron el cortisol en plasma, la tasa de excreción urinaria de 6 sulfato de melatonina (6-SM), la adrenalina, noradrenalina, frecuencia cardiaca, tensión arterial, así como la temperatura oral y registros de actividad del sueño.

El cortisol plasmático no mostró una perturbación de su patrón normal circadiano durante los dos turnos de noche consecutivos. Tuvo una tendencia a aumentar sus valores durante la primera parte de la segunda noche (inicio: emparejado $t=1,82 ; \mathrm{P}=0,047$; medio: emparejado $t=5,80 ; \mathrm{P}=0,005)$.

La excreción urinaria de 6-SM mostró su habitual patrón, con tasas significativamente más altas durante los turnos de noche $(\mathrm{F}=15,013 ; \mathrm{P}=0,000)$, sobre todo en la segunda mitad de la noche. No surgieron diferencias significativas que entre los dos turnos de noche, a pesar de una tendencia a la reducción de la tasa de excreción se observó en la segunda noche $(\mathrm{t}=1,926, \mathrm{P}=0,061)$.

La relación de noradrenalina/adrenalina no mostró diferencias significativas entre los primeros mitades de los cuatro turnos, mientras que fue significativamente mayor durante la segunda mitad de los turnos de noche (ANOVA: $F=62.89 ; \mathrm{p}<0.001$ ).

No surgieron diferencias significativas para la presión arterial o para la frecuencia cardíaca en reposo entre los turnos, aunque se registraron valores ligeramente más altos al principio de cada turno.

\section{Kecklund G. et al. ${ }^{14}$}

Evaluó el cambio de un sistema de tres turnos de rotación lenta ( 7 turnos seguidos) para una rotación rápida (tres o cuatro turnos seguidos) y examinó los efectos sobre el bienestar, la salud, el sueño y la satisfacción en el trabajo.

Durante dos años (1991-1993) fue seguida una población de 72 trabajadores masculinos de una industria de automoción, con medición de la presión arterial y lípidos en sangre, además de un cuestionario de los hábitos alimentarios.

Los resultados mostraron un aumento en la satisfacción con el nuevo horario de trabajo, menos problemas de sueño / vigilia y menos perturbaciones sociales causadas por el trabajo por turnos. Los lípidos de la sangre, aunque no se obtuvo significancia estadística, mostraron aumento del HDL y una relación LDL/HDL reducida en el examen de salud dos años después de la intervención.

\section{Bøggild H. et al. ${ }^{15}$}

En este estudio se introdujo una intervención cuasiexperimental controlado en 4 salas de hospital, siendo otras 6 salas utilizadas como control. Se dispuso de una población de 101 enfermeras y auxiliares de enfermería del condado de Jutlandia, Dinamarca, seguidas durante seis meses con mediciones de colesterol y triglicéridos. La estrategia analítica principal es examinar la relación entre la "exposición" a un cambio en la 
secuencia de turnos de trabajo y las modificaciones en los biomarcadores de riesgo cardiovascular.

En las distintas salas de intervención se plantearon distintos turnos ergonómicos con valoración del avance rápido de giro, regularidad y retraso en el inicio del turno de mañana. En el acuerdo alcanzado de la programación se respetaron los siguientes principios:

- Máximo de 3 a 4 turnos de noche consecutivos.

- Más horarios regulares y predecibles.

- El personal puede elegir la rotación.

- Reducir al mínimo el trabajo de fin de semana.

Las mediciones se valoraron mediante cuestionario al inicio, 6 y 12 meses con controles analíticos al inicio y a los 6 meses. Las valoraciones sanguíneas se realizaban al menos tras 72 horas del último turno de la noche.

El cambio ergonómico en la regularidad de los turnos fue asociado a una disminución en el nivel de triglicéridos y una disminución en el colesterol total. Con los cuatro principios acordados se produce una disminución del 8\% en el colesterol total, una disminución del 15\% en el colesterol LDL, un aumento del 9\% en el colesterol HDL.

\section{Nesthus T. et al. ${ }^{16}$}

El propósito de este estudio fue examinar el ritmo circadiano de la temperatura en el sentido de las agujas del reloj (CW) y en sentido antihorario (CCW) con secuencias de giro rápido de cambios.

Durante 3 semanas se sometió a 28 controladores aéreos de una compañía USA (12 hombres y 16 mujeres) divididos en dos grupos, a un turno a favor de las agujas del reloj y otro en contra, examinando el ritmo circadiano de temperatura.

Los resultados revelaron efectos significativos según el tipo de giro en la curva que describe el ciclo circadiano de la temperatura (amplitud F $(1,21)=14,62 ; \mathrm{p}<0,05$ y acrofase $\mathrm{F}(1,21)=4,43 ; \mathrm{p}<0,05)$. La amplitud para el giro a la izquierda $\left(\mathrm{M}=0,3{ }^{\circ} \mathrm{C}\right)$ fue significativamente menor que la amplitud de la rotación con giro en el sentido de las agujas del reloj $\left(\mathrm{M}=0,5^{\circ} \mathrm{C}\right)$ a través de ambos semana del trabajo por turnos. Además, un retardo de 84 minutos de la acrofase se encontró para la condición CCW (M = 18:08) relativa a la condición de $\mathrm{CW}(\mathrm{M}=16: 44)$ a través de las dos semanas del trabajo por turnos.

La amplitud reducida y la fase de retardo en acrofase para el grupo CCW pueden indicar una interrupción temporal del ritmo circadiano de la temperatura corporal central como una función de la condición de rotación de turnos.

\section{De Valck E. et al. $0^{17}$}

Mediante un simulador de conducción, examino la somnolencia subjetiva tras turnos de mañana, tarde y noche, así como el estrés objetivo mediante la medición del cortisol salival, en turnos de un avance rápido a favor de las agujas del reloj y un sistema de turno rotativo lento hacia atrás.

Este estudio comparativo de trabajo por turnos se llevó a cabo en una planta química en Bélgica, entre treinta y seis voluntarios varones, que se dividieron en dos grupos, permaneciendo una media de 3 años en el grupo de rotación lenta antihorario y 1 año en el nuevo sistema rotatorio rápido a favor de las agujas del reloj.

El nivel medio de cortisol salival después de la segunda mañana fue 4,91 mg / 1 para el grupo de rotación lenta y 4,85 mg / 1 para el grupo de rotación de avance rápido. Estos niveles de cortisol no difirieron significativamente entre los sistemas de rotación de desplazamiento $(\mathrm{F}(1,34)=0.009$; no significativa $)$. 
Las muestras salivales de cortisol, que reflejan la actividad del eje hipotálamopituitario-adrenal, tomadas al inicio de la semana laboral no diferenciaron significativamente entre el avance rápido y el sistema de turnos de rotación lenta hacia atrás.

\section{Viitasalo K. et al. ${ }^{18}$}

Realiza un estudio de intervención controlado para evaluar los efectos de un cambio en la rotación de turnos (dirección y velocidad) y un cambio en la flexibilidad de sistema de turnos en 84 trabajadores varones de mantenimiento de una compañía aérea de Finlandia.

Los efectos de los dos nuevos sistema de turnos diferentes en la salud se estudiaron mediante la comparación de los resultados de salud de 5 a 6 meses antes (octubrenoviembre de 2004) y de 7 a 8 meses después (noviembre-diciembre de 2005), de la aplicación del nuevo sistema de turnos.

De los 84 varones participantes, libres de cardiopatía isquémica al inicio del estudio, 40 de ellos cambió a un sistema de turnos con rotación rápida hacia delante, 22 cambió a trabajar en un sistema de turnos más flexible, y 22 persistieron en el sistema antiguo de turnos, y así se formaron los grupos de referencia.

Los trabajadores que se iniciaron en el sistema de turnos girando rápidamente hacia delante llevaban unos 10 años más de antigüedad que los que comenzaron en el sistema de turnos flexibles.

Los cambios en la presión arterial sistólica diferían entre los tres grupos de estudio ( $\mathrm{p}=0,049$ ). La presión arterial sistólica disminuyó $6 \mathrm{~mm} \mathrm{Hg}$ en el grupo con el sistema de turnos flexibles, se incrementó en $2,5 \mathrm{~mm} \mathrm{Hg}$ en el grupo con el sistema de desplazamiento rápido hacia adelante, pero se mantuvo sin cambios en el sistema antiguo.

Los cambios en la presión arterial diastólica fueron no significativos. Cuando se compararon los tres grupos de estudio, los cambios en la frecuencia cardíaca tenían una tendencia a la disminución $(\mathrm{P}=0: 06)$, y esta tendencia persistió cuando el sistema de cambio flexible, que en comparación con el sistema anterior en más análisis $(\mathrm{P}=0: 06)$.

El nuevo sistema de cambio no afectó a los otros factores de riesgo cardiovascular: la relación cintura-cadera, IMC, los lípidos en sangre, el control glucémico medido por HbA1c, la glucosa en ayunas.

\section{Estudios cohortes y casos controles}

\section{Morikawa Y. et al. ${ }^{19}$}

Este estudio investigó si el trabajo por turnos es un factor de riesgo para el desarrollo de la diabetes mellitus, para lo cual 2860 trabajadores masculinos japoneses de una fábrica de plásticos fueron seguidos durante 8 años (1994-2001). Los trabajadores fueron clasificados de cuello blanco y cuello azul. Se comparaban tres tipos de turnos: fijo diurno, rotatorio dos turnos (Mañanas-Noches), rotatorio tres turnos (Mañana-tardenoche, con giro hacia la izda.).

Entre los trabajadores de cuello azul, la tasa de incidencia era más alta para los trabajadores de dos turnos (6.84/1000 personas-año), seguidos por los trabajadores de tres turnos (5.32/1000 personas-años) y finalmente los trabajadores diurnos fijos (04:23 / 1.000 personas-año).

El riesgo relativo (RR) de diabetes mellitus para los trabajadores de dos turnos en comparación con los trabajadores fijos durante el día era de 1,70 (1,80 después del ajuste por edad, IMC y AF). Cuando se comparan con los empleados de tres turnos el RR es del 1.33, no variando con los diferentes ajustes, no alcanzándose significación estadística en ninguno de los dos grupos de turnos. Es de destacar que los riesgos relativos de los 
trabajadores de tres turnos comparados con los trabajadores fijos durante el día eran más pequeños que los de los trabajadores de dos turnos.

Cuando se utilizaron los trabajadores de cuello blanco en la misma fábrica como grupo de referencia, se encuentra un aumento estadísticamente significativo en el riesgo de diabetes mellitus por los trabajadores de dos turnos (RR 1,93). Este aumentó después de los correspondientes ajustes (RR 2,1 IC 95\% 1,00 a 4,34).

\section{Oyana l. et al. ${ }^{20}$}

El objetivo de su estudio era investigar el efecto del cambio de turnos de trabajo en el riesgo de desarrollar intolerancia a la glucosa. La población de estudio fue de 6413 empleados de una industria química japonesa cuyos horarios de trabajo se mantuvo constante en el período de seguimiento (media del período de seguimiento 23,2 años). Se comparaban tres tipos de turnos: turno fijo, rotatorio de 2 turnos (Mañanas-Noches) y rotatorio de 3 turnos (Mañana-Tarde-Noche).

Durante el tiempo de seguimiento de 23,2 años (63 601 personas-año en total, la media del período de seguimiento 9,9 años), se detectaron 1.209 incidencias de intolerancia a la glucosa.

En la cohorte original la tasa de incidencia de intolerancia a la glucosa era siempre mayor en el grupo de trabajadores con rotación de dos turnos. El OR fue significativamente mayor entre los trabajadores del turno de rotación frente a los trabajadores de día (OR 1,93 IC del 95\% 1,61 a 2,32 y OR 3,02 IC del 95\%: 2,50 a 3,66, para trabajadores de 3 y 2 turnos respectivamente). Los resultados no fueron alterados incluso significativamente después de posteriores ajustes.

El Hazard ratio ajustada por edad de dos turnos en comparación con los trabajadores de 3 turnos fue de 1,45 (IC del 95\%: 1,13 a 1,86).

\section{Estudios descriptivos transversales}

\section{Vangelova K. et al.21}

El objetivo de este estudio era ver el efecto de la dirección de rotación en las variaciones de cortisol, la fatiga y alteraciones del sueño en 25 ingenieros de sonido de Bulgaria.

Los ingenieros de sonido han sido seleccionados al azar. Se dividieron en dos grupos sobre la base de la dirección de rotación. El grupo con rotación giratoria hacia delante estaba compuesto por 13 ingenieros de sonido ( 4 hombres y 9 mujeres) de entre 45,1 \pm 7,3 años, con tiempo de servicio $21,4 \pm 11,3$ y otro grupo de rotación hacia atrás compuesto por 12 ingenieros de sonido ( 4 hombres y 8 mujeres) de edad de 51,7 $\pm 6,0$ años, con tiempo de servicio de $27,4 \pm 8,7$.

La prueba de Kolmogorov-Smirnov se utilizó para estudiar la distribución de cortisol salival y mostró una distribución normal $(\mathrm{P}>0,05)$. El cortisol retuvo el patrón diurno típico con valores altos en la mañana temprano y la secreción más baja durante el día.

El efecto de la dirección de rotación en la secreción de cortisol no alcanzó significación estadística, pero el efecto del cambio es altamente significativa $(\mathrm{F}(3,66)=$ 24,357, $\mathrm{p}=0,001)$ con valores de secreción de cortisol más altos en la mañana y en los turnos de noche. Además se encontró interacción significativa del sentido de giro ( $\mathrm{F}$ $(3,66)=6.046, p=0.001)$, con valores más altos de cortisol en el grupo de rotación hacia atrás.

Los datos mostraron mayor cortisol salival en el grupo de rotación hacia atrás en comparación con la rotación hacia delante. 


\section{Puttonen S. et al. ${ }^{22}$}

Estudio la asociación de síndrome metabólico (SM) entre extrabajadores a turnos. La población de estudio fue de 1811 trabajadores de una compañía aérea (de los cuales 1009 hombres), durante los años 2006-2008, dividiendo la población a estudio entre trabajadores con 2 turnos (Mañanas-Noches), nocturnos (Mañanas-Tardes-Noches), de día y de vuelo.

Entre los extrabajadores del turno de sexo masculino, los Mets (unidad de medida del índice metabólico) fueron más frecuentes comparando con los trabajadores masculinos de día. (IDF: OR 2,13 con IC 95\% 01,35; 3,37; NCEP: OR 1.83, $95 \%$ CI 1,13; 2,96). Las asociaciones no cambiaron después de los ajustes adicionales.

Los hombres 2 turnos tenían un riesgo elevado SM definidos IDF (OR 1.64, IC del 95\%: 1,06; 2,55), pero la asociación se debilitó en los análisis plenamente ajustadas (OR 0,93 IC 95\% 2,24; 1,48). Entre las mujeres, no se observaron diferencias significativas en la prevalencia de los Mets.

Este estudio mostró que entre los hombres, el ex trabajador por turnos se asocia con el aumento en la presentación de la FID y NCEP criterios de SM y que los hombres que trabajan en dos turnos tuvieron una tendencia al aumento de la prevalencia de los Mets, pero la asociación desapareció con posterior ajuste.

\section{Mauss D. et al. ${ }^{23}$}

Exploró la asociación entre los horarios de trabajo que implica turnos de noche y el síndrome metabólico y si estas asociaciones eran diferentes entre los que trabajan en 3 o 4 ciclos de turnos $\left(7^{1 / 4}\right.$ y 6 horas respectivamente).

La población de estudio fue de 765 trabajadores masculinos de una empresa industrial alemana, con una edad media de 36 años (desviación $\pm 9,63$ ) y con recogida de muestras durante el año 2007.

Para detectar importante diferencias en la salud relacionados con la frecuencia de rotaciones del turno de noche, se identificaron dos subgrupos a partir de los trabajadores que realizaban turnos de noche (Grupo B): B1 se componía de empleados con horarios de 3 turnos rotativo mientras B2 se componía de empleados con un horario rotativo 4 turnos.

En comparación con los trabajadores del turno de día $(\mathrm{N}=632)$, los empleados que trabajan en un ciclo de 3 turnos tenían mayores probabilidades de satisfacer la definición de síndrome metabólico (OR = 2,56 IC 95\% 1,38 a 4,75). Los empleados que trabajan ciclos de 4 turnos menos probabilidades de tener el síndrome metabólico (OR = 1,22 IC $95 \% 0,73$ a 2,05$)$ y tuvieron mayor actividad parasimpática medido por la variabilidad de la frecuencia cardíaca (OR = 2,20 IC 95\% 1,04 a 4,63).

\section{Estudio piloto}

\section{Katermann T. et al. ${ }^{24}$}

El objetivo de este estudio fue identificar el riesgo aterosclerótico utilizando la velocidad de onda del pulso (VOP) en trabajadores de acero en distintas rotaciones del trabajo por turnos. Los participantes son 77 trabajadores varones de una fábrica de acero Belga (32 CW rápido, $30 \mathrm{CCW}$ lento, 15 trabajadores de día), con una media de edad $42 \pm$ SD 7,6 años y con al menos 5 años de experiencia en su horario trabajo actual.

La velocidad de la onda del puso (VOP) de los tres grupos de trabajo no presentaron diferencias estadísticamente significativas. Estos resultados no cambiaron tras un nuevo ajuste de la prevalencia (sí/no) de los siguientes parámetros de salud: diabetes, enfermedad renal, la hipertensión y la hipercolesterolemia. En particular, se observó que los tres valores más altos valores de VOP $(10,3,10,4$ y 11,1 m/s, respectivamente) se producían exclusivamente en los turnos de trabajo $\mathrm{CW}$ rápidos. 


\section{DISCUSIÓN}

\section{Efectos de la intervención}

Los resultados de esta revisión bibliográfica ponen de manifiesto que la mayoría de los trabajos de intervención parecen tener efectos positivos o neutros sobre la salud. No se observó que ninguno pudiera ser considerado especialmente perjudicial para los trabajadores. Hay tres tipos de intervención que han concluido tener efectos beneficiosos: el cambio de rotación lenta a rotación rápida, cambio en la dirección de rotación hacia delante y la autoprogramación de los turnos.

Entre los factores que dificultan el análisis de la información y su posterior comparación de los estudios mencionados en esta revisión se encuentran:

- El efecto del trabajador sano.

- Múltiples definiciones de trabajo por turnos y patrones de funcionamiento o sistemas de rotación diferentes.

- Tamaños muestrales y tiempo de la exposición de las intervenciones.

- Categorización por grupo sectorial.

- Categoría profesional y nivel cultural de los trabajadores.

- Suceptibilidad individual de los trabajadores (búhos/ alondras).

- Los distintos tipos de intervención.

- Variedad de diferentes biomarcadores utilizados.

- Sesgo de atención o efecto Hawthorne.

A continuación clasificaremos los trabajos revisados en función de la intervención aplicada:

\section{Cambios de dirección de rotación}

Consiste en realizar turnos hacia delante o a favor de las agujas del reloj (MañanaTarde-Noche), y hacia atrás o sentido antihorario (Mañana-Noche-Tarde // Noche-TardeMañana).

Tres de los trabajos ${ }^{12,13,21}$ se engloban dentro de este grupo analizando a su vez distintos biomarcadores. El cortisol salival es analizado por Vangelova K. et al. ${ }^{21}$, produciéndose un aumento significativo del mismo en el turno con rotación antihoraria, cuestión que no se reproduce en el trabajo de Costa G. et al. ${ }^{13}$, el cuál utilizó el cortisol plasmático como biomarcador de estrés. En este trabajo además, Costa analizó la adrenalina y noradrenalina, con aumento de la misma en sentido antihorario, cuestión que coincide con el estudio de Orth-Gomer $\mathrm{K}$ et al. ${ }^{12}$, en el que además comprobó una disminución de la glucosa, triglicéridos, ácido úrico y TAS en sentido horario. El resto de los biomarcadores analizados no se modificaron con la dirección de rotación.

Lo anteriormente expuesto está acorde con estudios epidemiológicos y de laboratorio que han sugerido que un cambio en la dirección de atrás hacia adelante en la rotación es más acorde con el ritmo circadiano del cuerpo, y que por tanto se puedan derivar efectos positivos para la salud ${ }^{25-27}$.

\section{Cambios en la velocidad de rotación}

Se dividen en turnos de rotación lenta, que rotan semanalmente; esto es, una semana de trabajo nocturno sigue un turno semanal de trabajo de tarde y, a continuación un turno de mañana. En el sistema de rotación rápida, sólo se trabajan uno, dos o, como mucho, tres días consecutivos en cada turno. 1-3 mañanas 1-3 tardes 1-3 noches. 
El único trabajo que se centra en este tipo de intervención (Kecklund), muestra una disminución del LDL/HDL y aumento del HDL, coincidiendo con el trabajo de Boggid (que analiza además otras intervenciones).

Clásicamente el sistema de turnos impuestos en EEUU favorecía los turnos con cambios de velocidad de rotación lenta y hacia atrás, para así adaptar mejor el trabajador a la noche. Esta postura no es secundada por el sistema europeo que prefiere rotaciones cortas para que el trabajador se adapte lo antes posible al horario normal diurno. El Instituto Nacional para la Seguridad y Salud Ocupacional (NIOSH), ha informado de la evidencia a favor de la velocidad de rotación rápida, pero muchas de las cadenas de producción difícilmente podrían adaptarse a esta cadencia de turnos ${ }^{28}$.

\section{Cambios en la dirección y velocidad de rotación}

Consiste en una combinación de los dos modelos expuestos anteriormente.

Nesthus T. et al. ${ }^{16}$ observó que la temperatura de los trabajadores CCW frente a los CW tenían la amplitud reducida y una fase de retardo en acrofase, lo que puede indicar una interrupción temporal del ritmo circadiano.

De Valk E. et al. ${ }^{17}$ no encontró diferencias en el cortisol salival cuando comparaba un turno de $\mathrm{CW}$ rápido y un CCW lento.

Vitasalo K. et al. ${ }^{18}$ estudió varios biomarcadores encontrando un aumento de la TAS en el grupo de rotación rápida hacia delante, con normalidad en el resto (relación cintura cadera, IMC, lípidos, glucosa, HbA1c).

Katerman T. et al. ${ }^{24}$ no encontró diferencias al medir la onda venosa del pulso al comparar un $\mathrm{CW}$ rápido con $\mathrm{CCW}$ lento.

La diversidad de intervenciones así como la de los biomarcadores empleados dificulta la extrapolación de estos datos, pero se puede objetivar que en todos los cambios con rotaciones rápidas hacia la derecha, ningún grupo obtuvo aumentos en los biomarcadores a excepción de la TAS en uno de los estudios.

\section{Cambios ergonómicos en los turnos}

En este tipo de intervención se aplican una serie de criterios ergonómicos a diferentes turnos (flexibilidad, rotación hacia delante, no más de 3 turnos consecutivos de noche, autoprogramación, etc.).

Bøggild $\mathrm{H}$. et al. ${ }^{15}$ elabora distintos turnos en función de diferentes criterios ergonómicos comprobando que se produce un aumento del HDL y disminución del LDL y de la fracción Colesterol total/HDL en el grupo con más criterios ergonómicos, lo cual está en consonancia con los resultados de las intervenciones planteadas por Orth-Gomer K. et al. ${ }^{12}$ y Kecklund G. et $\mathrm{al}^{14}$.

Vitasalo K. et al. ${ }^{18}$ valorando los turnos flexibles encontró como resultado la disminución de la TAS y FC.

Se comprueba que son posibles programaciones más saludables, con criterios ergonómicos ya planteados por otros autores ${ }^{25-27}$.

\section{Cambios en el número de turnos}

En este apartado se comparan dos, tres o cuatro turnos y extrabajadores a turnos. Cuando se comparan dos con tres turnos nos referimos a trabajadores que realizan mañanas y noches (dos turnos), y mañanas, tardes y noches (3 turnos), difiriendo en la velocidad y dirección de rotación. Tres y cuatro turnos hacen referencia a horarios de trabajo al día (7 horas/3 veces al día o 6 horas/4 veces al día).

La diabetes mellitus es analizada en dos estudios japoneses ${ }^{19-20}$ que comparan ciclos de dos turnos frente a tres turnos en sentido antihorario, encontrando ambos un aumento 
de diabetes en los que realizan dos turnos frente a los que realizan tres. En uno de ellos, Morikawa Y. et al. ${ }^{19}$, subdividió la muestra de trabajadores por categoría profesional en trabajadores de cuello blanco y cuello azul, encontrando un aumento de DM en ambas categorías en los trabajadores de dos turnos.

El síndrome metabólico es analizado por dos trabajos. Puttonen S. et al. ${ }^{22}$ muestra que hay un aumento de síndrome metabólico entre los extrabajadores a turnos. Mauss D. et $a .^{23}$, manifiesta que hay aumento de síndrome metabólico entre los trabajadores de 3 turnos en dirección antihorario frente a los de 4 turnos ( 7 horas frente a 6 ).

Existen dos estudios en el campo de los genes reloj que dan base fisiológica para explicar estos hechos. En uno de ellos, modificaciones en genes reloj ${ }^{29}$ de ratas, revelaron que eran hiperfágicas y obesas, con tendencia a desarrollar síndrome metabólico con hiperlipidemia, esteatosis hepática e hiperglucemia, con una producción insuficiente de insulina compensatoria, un sello distintivo de la diabetes mellitus tipo 2 . El otro experimento demuestra, lo que sugiere una posible interacción de los transportadores de la serotonina ${ }^{30}$ y la variación del gen CLOCK en la susceptibilidad genética a desarrollar síndrome metabólico en la rotación de los trabajadores por turnos.

Como consecuencia de lo anteriormente expuesto hemos elaborado las siguientes conclusiones:

- Los artículos publicados sugieren un impacto del trabajo por turnos en los factores de riesgo cardiovascular.

- El cambio de la dirección de los turnos de atrás hacia adelante puede derivar efectos positivos sobre los factores de riesgo cardiovascular.

- Turnos ergonómicamente diseñados disminuyen las alteraciones lipídicas, TA y FC.

- Rotaciones rápidas en sentido a las agujas del reloj pueden ser beneficiosas o menos lesivas.

- Trabajos a dos turnos (mañana-noche) frente a tres turnos (mañana-tarde-noche), parecen ser potencialmente más favorecedores de diabetes mellitus y síndrome metabólico.

- Estudios experimentales con genes reloj demuestran que su modificación produce alteraciones en los hidratos de carbono y en el metabolismo de los lípidos.

Teniendo en cuenta que no hay un sistema óptimo de turno único que se pueda utilizar en la industria o el comercio en todos los lugares de trabajo, sin embargo, hay sistemas de turnos que son más favorables, y es en este contexto en el que realizamos las siguientes recomendaciones:

1. Los turnos de noche deben reducirse lo más posible. Si esto no es posible, los sistemas de turnos rotativos de forma rápida son preferibles a las que giran lentamente. El Trabajo nocturno permanente no parece ser recomendable para la mayoría de los trabajadores por turnos.

2. Jornadas de trabajo prolongadas (9-12 horas), sólo deberían ser consideradas cuando la naturaleza del trabajo y la carga del mismo son adecuados, además de que el sistema de turnos se haya diseñado para reducir al mínimo la acumulación de fatiga y la exposición a tóxicos.

3. Un comienzo temprano para el turno de la mañana debe ser evitado. Los horarios de trabajo flexibles se pueden conseguir en todos los sistemas de turnos.

4. Cambios rápidos (por ejemplo, de turno de noche a turno de la tarde en el mismo día), deben ser evitados. El número de días consecutivos de trabajo debe limitarse a cinco y siete. Cada sistema de turnos debería incluir algunos fines de semana libres con al menos dos días consecutivos de descanso. 
5. La rotación hacia adelante (la rotación en sentido horario: mañana/tarde/noche) parecen ser los más adecuados.

6. La elección de los turnos será discutida con los interesados, sobre la base de una información completa y precisa que permita tomar decisiones de acuerdo con las necesidades individuales.

7. Desarrollar un plan de educación sanitaria a fin de explicar a los interesados/as los principios de una alimentación sana y estilos de vida saludable.

8. Establecer un sistema de vigilancia de la salud que permita detectar rápidamente problemas de adaptación y problemas de salud asociados al trabajo a turnos, que nos permitan intervenir de manera preventiva antes de que aparezcan daños irreversibles.

\section{REFERENCIAS BIBLIOGRÁFICAS}

1. Consejo CEE. Directiva 2003/88/CE del Parlamento Europeo y del Consejo de 4 de noviembre de 2003 relativa a determinados aspectos de la ordenación del tiempo de trabajo [Internet]. 2003. Recuperado a partir de: http://eur-lex.europa.eu/LexUriServ/LexUriServ.do?uri=OJ: L:2003:299:0009:0019: ES: PDF.

2. INSHT. VII Encuesta Nacional de Condiciones de Trabajo de 2011 [Internet]. 2011. Recuperado a partir de: http://www.insht.es/InshtWeb/Contenidos/Documentacion/FICHAS\%20DE\%20PUBLICACIONES/ EN\%20CATALOGO/OBSERVATORIO/Informe\%20\%28VII\%20ENCT\%29.pdf.

3. P. Cazamian. Night Work - Its effects on the health and welfare of the worker [Internet]. OIT;1997. Recuperado a partir de: http://www.ilo.org/public/spanish/standards/relm/ilc/ilc89/rep-iii1b-c1.htm\#_ftn4.

4. WHO. OMS | Enfermedades cardiovasculares [Internet]. WHO. [citado 19 de enero de 2014]. Recuperado a partir de: http://www.who.int/mediacentre/factsheets/fs317/es/.

5. Frost P, Kolstad HA, Bonde JP. Shift work and the risk of ischemic heart disease - a systematic review of the epidemiologic evidence. Scand J Work Environ Health. mayo de 2009;35(3):163-79.

6. Mosendane T, Mosendane T, Raal FJ. Shift work and its effects on the cardiovascular system. Cardiovasc J Afr. agosto de 2008;19(4):210-5.

7. Esquirol Y, Perret B, Ruidavets JB, Marquie JC, Dienne E, Niezborala M, et al. Shift work and cardiovascular risk factors: new knowledge from the past decade. Arch Cardiovasc Dis. diciembre de 2011;104(12):636-68.

8. Vyas MV, Garg AX, Iansavichus AV, Costella J, Donner A, Laugsand LE, et al. Shift work and vascular events: systematic review and meta-analysis. BMJ. 2012;345: e4800.

9. Knutsson A. Health disorders of shift workers. Occup Med Oxf Engl. marzo de 2003;53(2):103-8.

10. Puttonen S, Härmä M, Hublin C. Shift work and cardiovascular disease - pathways from circadian stress to morbidity. Scand J Work Environ Health. marzo de 2010;36(2):96-108.

11. Informe final: reunión tripartita de expertos sobre la ordenación del tiempo de trabajo [Internet]. Ginebra: OIT; 2011 oct. Report No.: ISBN: 978-92-2-325411-7 (web pdf). Recuperado a partir de: http://www.ilo. org/travail/whatwedo/eventsandmeetings/WCMS_210831/lang--en/index.htm.

12. Orth-Gomér K. Intervention on coronary risk factors by adapting a shift work schedule to biologic rhythmicity. Psychosom Med. octubre de 1983;45(5):407-15.

13. Costa G, Ghirlanda G, Tarondi G, Minors D, Waterhouse J. Evaluation of a rapidly rotating shift system for tolerance of nurses to nightwork. Int Arch Occup Environ Health. 1994;65(5):305-11.

14. Kecklund G, Statens institut för psykosocial miljömedicin. Omläggning av skiftschema: konsekvenser för välbefinnande, hälsa och arbetstrivsel. Resultatrapport 2, Resultatrapport 2,. Stockholm: Statens institut för psykosocial miljömedicin; 1994.

15. Bøggild H, Jeppesen HJ. Intervention in shift scheduling and changes in biomarkers of heart disease in hospital wards. Scand J Work Environ Health. abril de 2001;27(2):87-96.

16. Nesthus T, Cruz C, Boquet A, Detwiler C, Holcomb K, Della Rocco P. Circadian temperature rhythms in clockwise and counter-clockwise rapidly rotating shift schedules. J Hum Ergol (Tokyo). diciembre de 2001;30(1-2):245-9.

17. De Valck E, Quanten S, Berckmans D, Cluydts R. Simulator driving performance, subjective sleepiness and salivary cortisol in a fast-forward versus a slow-backward rotating shift system. Scand J Work Environ Health. febrero de 2007;33(1):51-7.

18. Viitasalo K, Kuosma E, Laitinen J, Härmä M. Effects of shift rotation and the flexibility of a shift system on daytime alertness and cardiovascular risk factors. Scand J Work Environ Health. junio de 2008;34(3):198-205. 
19. Morikawa Y, Nakagawa H, Miura K, Soyama Y, Ishizaki M, Kido T, et al. Shift work and the risk of diabetes mellitus among Japanese male factory workers. Scand J Work Environ Health. junio de 2005;31(3):179-83.

20. Oyama I, Kubo T, Fujino Y, Kadowaki K, Kunimoto M, Shirane K, et al. Retrospective cohort study of the risk of impaired glucose tolerance among shift workers. Scand J Work Environ Health. julio de 2012;38(4):337-42

21. Vangelova K. The effect of shift rotation on variations of cortisol, fatigue and sleep in sound engineers. Ind Health. octubre de 2008;46(5):490-3.

22. Puttonen S, Viitasalo K, Härmä $M$. The relationship between current and former shift work and the metabolic syndrome. Scand J Work Environ Health. julio de 2012;38(4):343-8.

23. Mauss D, Litaker D, Jarczok MN, Li J, Bosch JA, Fischer JE. Anti-clockwise rotating shift work and health: would you prefer 3-shift or 4-shift operation? Am J Ind Med. mayo de 2013;56(5):599-608.

24. Kantermann T, Duboutay F, Haubruge D, Kerkhofs M, Schmidt-Trucksäss A, Skene DJ. Atherosclerotic risk and social jetlag in rotating shift-workers: first evidence from a pilot study. Work Read Mass. 1 de enero de 2013;46(3):273-82.

25. Knauth P. The design of shift systems. Ergonomics. marzo de 1993;36(1-3):15-28.

26. Knauth. Speed and direction of shift rotation. J Sleep Res. diciembre de 1995;4(S2):41-6.

27. Sallinen M, Kecklund G. Shift work, sleep, and sleepiness - differences between shift schedules and systems. Scand J Work Environ Health. marzo de 2010;36(2):121-33.

28. CDC - Publicaciones de NIOSH - El trabajo por turnos en lenguaje sencillo (97-145) [Internet]. [citado 20 de enero de 2014]. Recuperado a partir de: http://www.cdc.gov/spanish/niosh/docs/97-145_sp/.

29. Turek FW, Joshu C, Kohsaka A, Lin E, Ivanova G, McDearmon E, et al. Obesity and metabolic syndrome in circadian Clock mutant mice. Science. 13 de mayo de 2005;308(5724):1043-5.

30. Sookoian S, Gianotti TF, Burgueño A, Pirola CJ. Gene-gene interaction between serotonin transporter (SLC6A4) and CLOCK modulates the risk of metabolic syndrome in rotating shiftworkers. Chronobiol Int. julio de 2010;27(6):1202-18

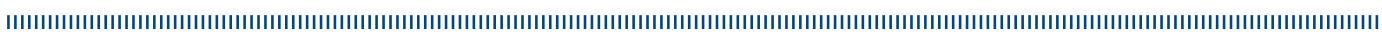

